\title{
Temporal variability of the seeing of TMT sites
}

T. Travouillon, S. G. Els, R. L. Riddle, M. Schöck, A. W. Skidmore, et al.

T. Travouillon, S. G. Els, R. L. Riddle, M. Schöck, A. W. Skidmore, E. Bustos, J. Seguel, D. Walker, J. Vasquez, R. Blum, P. Gillett, B. Gregory, "Temporal variability of the seeing of TMT sites," Proc. SPIE 7012, Groundbased and Airborne Telescopes II, 701220 (10 July 2008); doi: 10.1117/12.787136

Event: SPIE Astronomical Telescopes + Instrumentation, 2008, Marseille, France 


\title{
Temporal variability of the seeing of TMT sites
}

\author{
T. Travouillon*a, S.G. Els ${ }^{\mathrm{b}}$, R.L. Riddle ${ }^{\mathrm{a}}$, M. Schöck ${ }^{\mathrm{a}}$, A.W. Skidmore ${ }^{\mathrm{a}}$, E. Bustos ${ }^{\mathrm{b}}$, J. Seguel $^{\mathrm{b}}$, D. \\ Walker ${ }^{b}$, J. Vasquez ${ }^{b}$, R. Blum ${ }^{b}$, P. Gillett, B. Gregory ${ }^{b}$ \\ ${ }^{a}$ TMT Observatory Corporation, 2632 E. Washington Blvd, Pasadena, CA 91107, USA \\ ${ }^{\mathrm{b}}$ Cerro Tololo Inter-American Observatory, Casilla 603, La Serena, Chile
}

\begin{abstract}
Seeing stability is an important criterion of site characterization. Two sites, with the same seeing statistics, could in principle differ in their temporal stability and hence have their observatories perform differently. Temporal variability can, however, be defined in several ways, all of which may determine the performance of the observatories in different manner. In this paper, we propose three methods to measure variability each focusing on different applications: Selection (maximization of observation time), Image quality (seeing variation within a given integration time) and finally Scheduling (prediction of seeing fluctuation on a given time scale). We apply these methods to the seeing of the TMT candidate sites to determine their stability properties.
\end{abstract}

Keywords: atmospheric turbulence, site testing, ELTs

\section{INTRODUCTION}

In astronomical site characterization, one of the least studied parameter is seeing variability. This is surprising since the seeing is often considered the parameter that has the biggest impact on telescope performance. Two sites, with the same seeing, could in principle differ in stability and hence have their observatories perform differently. Being an atmospheric quantity, the seeing has a log-normal distribution. To first order, two sites with the same median seeing can therefore be compared using the FWHM of their distribution to discriminate them based on variability. A better job can however be made by defining quantitatively how the variability affects the observatory. To our knowledge, the issue of seeing variability has only been addressed by Racine [1] and Vernin et al. [2]. These studies were limited to one site and one aspect of the variability. Their goal was to determine a characteristic time at which the seeing reaches a given variability. Seeing variability has, however, more than one effect on observations and can therefore be defined differently according to which aspect of the observation needs to be optimized. For example, seeing variability manifests itself at the scheduling level, as different observations have different seeing requirement.

Three methods are proposed in this document, each focused on a different aspect of the variability and its application to a telescope project. These applications are:

1. Selection: Maximization of observation time under a given seeing criterion. New observatories have an estimated breakdown of the percentage of time the telescope will be used on a given science goal. Each of these goals needs specific observing conditions. This method calculates the total amount of time the telescope will be able to observe within these conditions.

2. Image quality: Seeing variation within a given integration time. The quality of the PSF depends on the seeing conditions averaged over the integration time of the observation. This method quantifies this effect by calculating the variance of the seeing within different time scales typical of astronomical observations.

3. Scheduling: Prediction of seeing fluctuations on a given time scale. This method, adopted from the previously quoted references, calculates in relative and absolute terms the typical time scale of the variability of the seeing. It offers a statistical idea of how different the seeing will be at a later time from the current seeing conditions.

*tonyt@caltech.edu

Ground-based and Airborne Telescopes II, edited by Larry M. Stepp, Roberto Gilmozzi, Proc. of SPIE Vol. 7012, 701220, (2008) · 0277-786X/08/\$18 · doi: 10.1117/12.787136 
These methods will be explained in detail and used to compare the variability of the seeing of the TMT candidate sites, namely Armazones, Tolar and Tolonchar which are the three Chilean sites evaluated by TMT in Chile, San Pedro Mártir which is located in Baja California in Mexico, and 13 North on Mauna Kea. More details about the sites can be found in Schöck et al. [3]

\section{ANALYSIS METHOD}

The three methods of analysis which are described in this paper are applied to the data of all five TMT sites. The actual amount of data varies for each site and we include all the data available up to May $1^{\text {st }}, 2007$. The objective is not only to find a metric to compare the sites variability, but also to make the metric quantitatively usable for different aspect of project performance.

Sites with better median seeing are expected to be better in this respect. However, the causes for large seeing values may be different for each site (e.g. thermals in the ground layer, jet stream at high altitude) and therefore may not occur at the same frequencies. Moreover, several sites have very similar seeing and one way to differentiate them may be their seeing stability.

The seeing dependence of the variability can be removed by normalizing it. The analysis of two of the three methods presented includes both absolute variability, expressed in arc seconds, and normalized variability, expressed as a percentage.

\section{Selection method}

The first temporal analysis method is oriented toward the practical aspects of observing with TMT. Once installed at one of the candidate site, the telescope performance will partially depend on the variations of the seeing within the integration time of an observation. Most observations require a given PSF in order to achieve their goal. A good selection criterion is therefore to choose a site with the largest fraction of observable time per year below a certain seeing within a usable integration time.

For each site, only seeing data that are within an interrupted time series longer than two hours are used in this method. This choice was made because interruptions in the measurements (caused by technical problems or clouds) may miss fast seeing fluctuations which, if discarded, can bias the data.

Using this selected data set, an integration time is chosen and the total amount of time that is at least as long as the integration and continuously under a given seeing value is added up. The total time found to satisfy these conditions is then divided by the total length of the data set in order to express it as a percentage of observable time. This percentage is the figure of merit which is measured for a range of integration time (from 10 to 60 minutes) and seeing values (from 0.3 to 1 arc seconds). We may choose, for example, to prefer a site which has the largest fraction of its observable time below 0.5 " for at least $20 \mathrm{~min}$ uninterrupted.

\section{Image quality method}

This method, known as pooled variance, is already used in astronomy (see [4]). The image quality or PSF is affected by the variability of the seeing within the integration time of the observation.

To compute the pooled variance, we first divide the time series of seeing data into bins of specific lengths and compute for each of these bins the variance. Only bins containing a sufficient amount of data were taken into account. The pooled variance is then the ensemble average over all these variance values and indicates the variation of the time series on the specific time scale. The pooled variance tells us the variance of the seeing within a given observation time. Here, we have assessed how the seeing of each site varies on time scales between 10 and $60 \mathrm{~min}$.

This method is also presented in a normalized way in order to provide a seeing independent metric. In this case, the variance calculated from each bin is divided by the mean of the seeing squared within the bin, making the variance unitless. 


\section{Scheduling method}

The fractional difference method was introduced by Racine [1]. This method works on time scales shorter than a night of observation and is practical because it does not require a constant and unbroken streak of measurements. The fractional difference is calculated for each night and averaged over the entire data set following the equation:

$$
f(\tau)=2 \times \frac{|\varepsilon(t+\tau)-\varepsilon(t)|}{\varepsilon(t+\tau)+\varepsilon(t)}
$$

where $\varepsilon$ is the seeing and the fractional difference is calculated for values of $\tau$ ranging from 1 to 60 minutes with 1 minute resolution. While the pooled variance tells how the seeing varies within a given time scale, the fractional difference tells us the expected difference in seeing between now and a later time, $\tau$, either expressed in absolute term (in arcseconds) by omitting the denominator from Eq.1, or normalized (in percentage). This method can help scheduling observations as it tells the characteristic time of the seeing stability.

\section{RESULTS}

The seeing measures which of all TMT sites, which are analyzed here are taken with a DIMM located on a tower $7 \mathrm{~m}$ above the ground. Figure 1 shows a comparison of the "selection method" for the seeing at all sites, respectively for integration times of $30 \mathrm{~min}$ and $60 \mathrm{~min}$. With this method, Tolonchar is the most stable site although it is indistinguishable from the other two Chilean sites in good seeing conditions. In both cases, Tolar and Armazones perform very similarly. This is an expected result as the two sites have similar mesoscale properties such as the type of terrain and the similar latitudes. Among the northern sites, Mauna Kea is more stable, giving away $10 \%$ to $20 \%$ of observation time of the Chilean sites above median seeing conditions. San Pedro Mártir drops another few percents of observation time in most of the seeing range but is similar to Mauna Kea on the 60min time scale where mesoscale effects become more important. It must be noted that this method does not decouple the seeing variability from the site's absolute seeing. This is not a normalized method and the sites with better seeing have, as expected, a larger fraction of continuously low seeing values. What this method provides is a new way to compare sites. It combines both, the absolute seeing and its variability, in one parameter which can directly be related to the expected observatory operations.
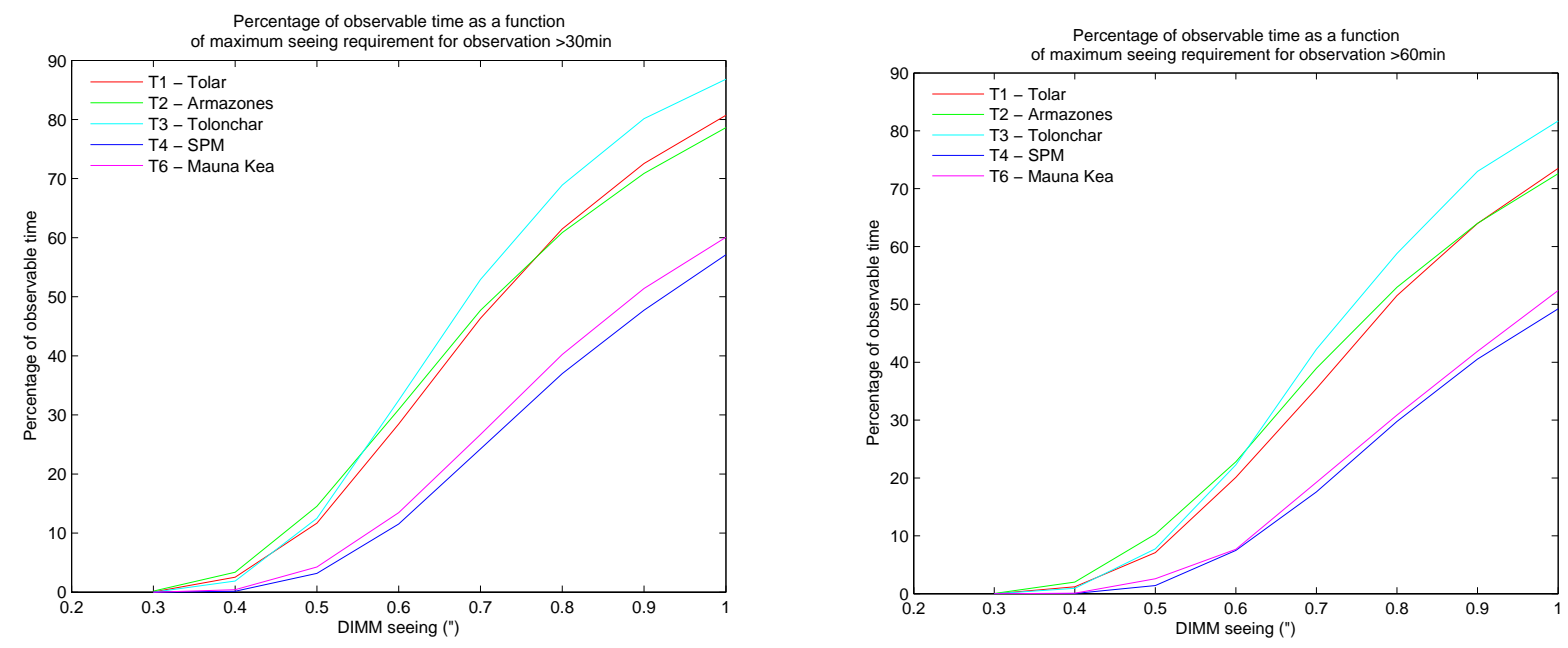

Fig.1. Fraction of total time below a given seeing for continuous integration times of 30min (left) and 60min (right).

The results of the "image quality" method are shown in Fig. 2 in absolute and normalized fashion. The absolute variability gives a comparison of the sites, again correlated with the seeing amplitude of each site. The noticeable 
difference from the previous method is the differentiation between Mauna Kea and San Pedro Mártir. Tolar and Armazones are indistinguishable while Tolonchar's variance is up to a factor of two lower.

Using the normalized version of this method, most of the differences between the sites decrease to the point where only Tolonchar appears as significantly better across the entire range of time scales. Previously the most variable site, San Pedro Mártir is now comparable with Tolar and Armazones and slightly better than Mauna Kea. It can be noted that Tolonchar has the most constant wind direction. A vast majority of the time, the wind blows from the Salar de Atacama, a large and flat desert with no outstanding terrain feature that could perturb the laminar flow. The other sites on the other hand, have a more variable wind rose. Depending on the wind direction, the local and mesoscale topography, will affect the seeing differently, hence increasing its temporal variability.
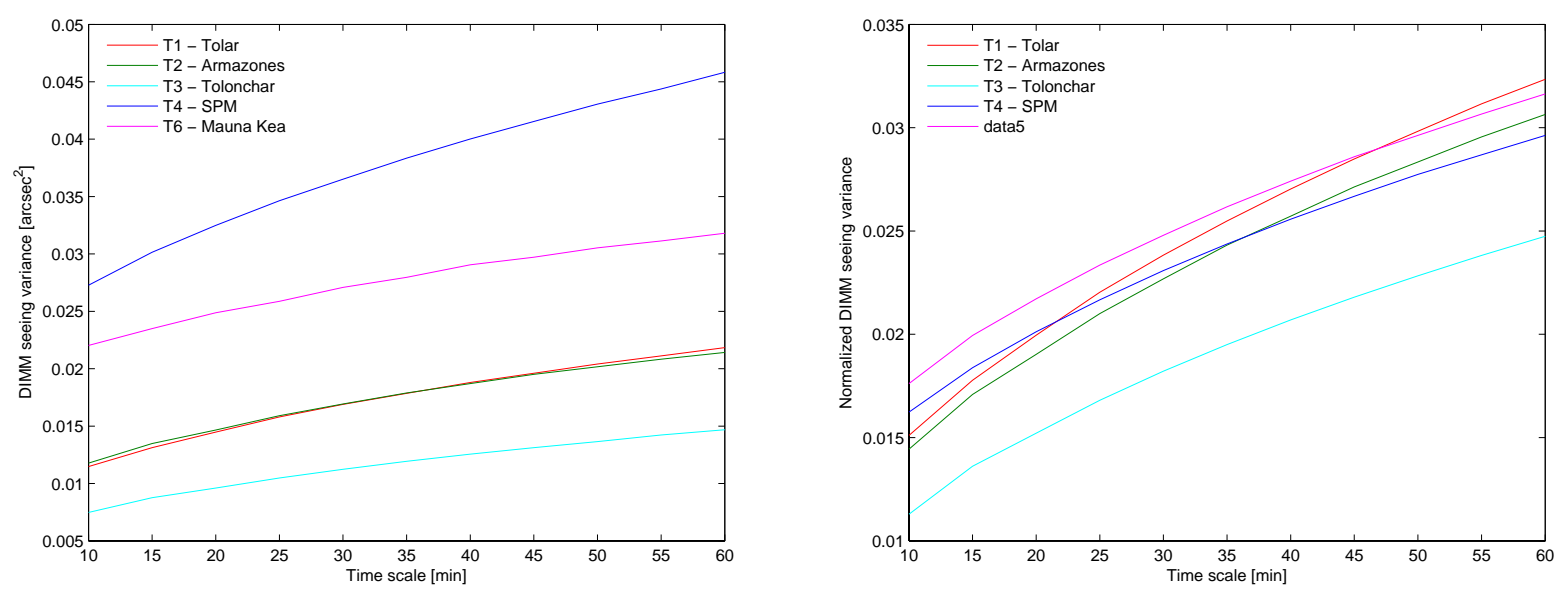

Fig.2. Pooled variance of the seeing of all sites over time scales up to an hour. The plot on the right is normalized.

The Pooled variance can also show some interesting details on time scales larger than typical observations. With the TMT site testing database consisting of over 2 years of seeing statistics for each site, it is possible to calculate the pooled variance for periods up to a year. As shown in Fig. 3, the sites' variability increases relatively smoothly over increasing time scales. The three Chilean sites however level very quickly at periods larger than a few days. This is not surprising considering the much smaller seasonal changes that they encounter. Tolar, for example, has the smallest temperature difference between summer and winter and no noticeable increase in variability for periods over a month long. The northern sites on the other hand have very marked winters and show an increase in variability on the season time scale. They also have an interesting increase on the 1 to 3 day scale. The reason for this increase is currently being looked into and is likely to be caused by local environmental parameters such as wind and heat fluxes.

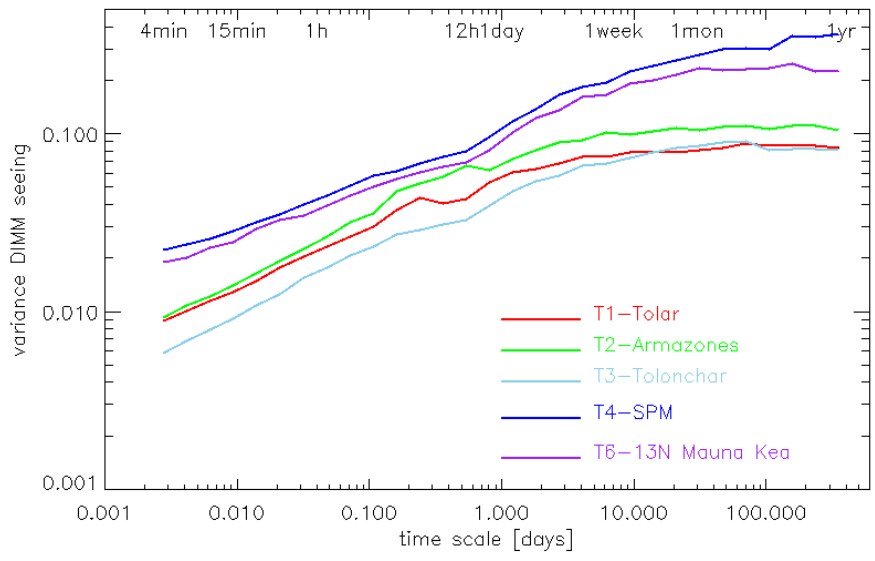

Fig.3. Pooled variance of the seeing over periods of up to a year. 
Fig. 4 shows the results of the "scheduling method", again in both absolute and normalized units. The results are similar to the previous method with sites ranking comparatively on all time scales. While this method tells us how different the seeing will be up to an hour from a given point, it cannot be used to discriminate between seeing improvements and deterioration. Adjacent seeing measurements (the TMT DIMM measures the seeing approximately every 1.5 minutes) show typical variations of 8 to $12 \%$ depending on the site. This variation increases by only another $10 \%$ after a full hour. The acceptability of these variations depends on the seeing requirement of the scheduled science. In Racine [1], who introduced this method, the author was able to fit the variability function and deduce a decay time defined by the speed of the seeing variation. Unfortunately our data did not fit the same function and no single number that defines the site variability was found. Instead, the three methods presented give a more flexible definition which can be adapted to the need of the observer. These methods can also be adapted to measure the variability of other parameters of the sites such as the isoplanatic angle or the coherence time.
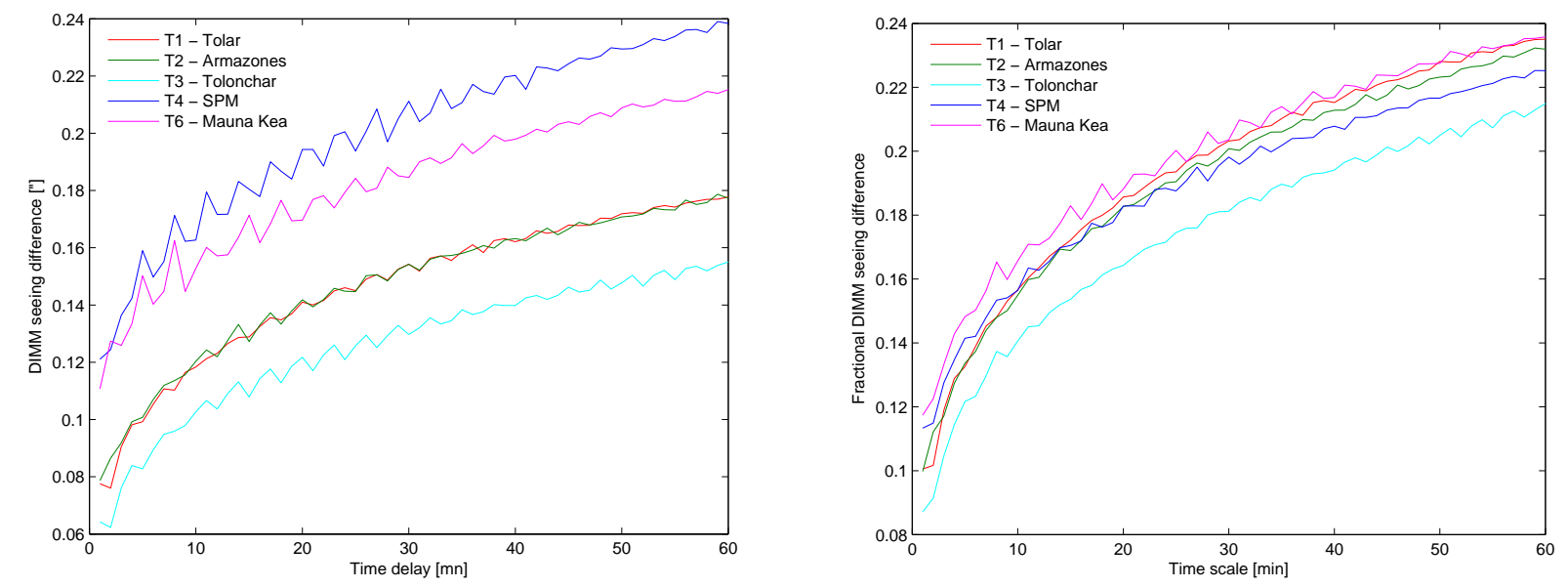

Fig. 4. Variation of the seeing over the time scale of one hour averaged over the entire data set. The right hand plot is normalized.

\section{CONCLUSION}

Three methods of variability measurement have been presented and illustrated using the seeing of the 5 TMT candidate sites. Comparing the sites in absolute terms only confirmed that sites with better seeing also varied less. However, when normalized by their respective seeing, we are able to differentiate sites which have the same median seeing. The value of this study also goes beyond the ranking of the sites with respect to variability. Understanding how quickly the seeing changes at a site allows for better use of the telescope time by providing more educated scheduling and maximizing the image quality of the different observations.

\section{AKNOWLEDGEMENT}

The authors gratefully acknowledge the support of the TMT partner institutions. They are the Association of Canadian Universities for Research in Astronomy (ACURA), the California Institute of Technology and the University of California. This work was supported as well by the Gordon and Betty Moore Foundation, the Canada Foundation for Innovation, the Ontario Ministry of Research and Innovation, the National Research Council of Canada, the Natural 
Sciences and Engineering Research Council of Canada, the British Columbia Knowledge Development Fund, the Association of Universities for Research in Astronomy (AURA) and the U.S. National Science Foundation.

\section{REFERENCES}

[1] Racine, R., "Temporal fluctuations of atmospheric seeing", PASP 108:372-374, (1996)

[2] Vernin, J., Munoz-Tunon, C., "The temporal behavior of seeing", New Astronomy reviews, 42, 6-8:451:454 (1998)

[3] Schöck, M., et al., "Status of the Thirty Meter Telescope Site Selection Program", These proceedings

[4] Dobson, A.K., Donahue, R. A, Radick, R. R., Kadlec, K. L., "Variance components in CA II H+K time-series observations", Proceedings of the 6th Cambridge Workshop, Seattle, WA, Sept. 18-21, 1989 San Francisco, CA, PASP, p. 132-135 (1990) 